\title{
Perioperative point-of-care ultrasound and emergency obstetric anesthesia
}

\begin{abstract}
This current review appraises the potentials of point-of-care ultrasound (POCUS) knowledge as a useful non-invasive tool in obstetric anesthetic management especially in emergent and difficult situations. The recent growth in the use of bedside ultrasound expands its diagnostic and therapeutic applications that encourage its use as a standard practice in anesthesia. Adverse events during pregnancy are infrequent, but may occur all of sudden. Availability of Ultrasound as a simple, non-invasive, non-ionizing diagnostic tool at the bed-side if complications occur helped to develop the concept of point-of-care ultrasound. This review covers the use of ultrasound in variable medical situations including, difficult tracheal intubation, and the risk of 'can't intubate, can't ventilate', is frequent in pregnant female. Point-of-care ultrasound of the airway permits detection of the cricothyroid membrane accurately, allowing rapid and safe surgical interference through front-of-neck airway access. As well as stomach contents can be identified by Gastric ultrasound that increases the risk for pulmonary aspiration in women. Pre-procedural ultrasound survey of the lumbar spine offers better accuracy and makes neuraxial techniques easier than traditional method. Finally both cardiac and lung ultrasound can distinguish between variable causes of circulatory failure in the pregnant patient, and guide management of such condition. As the importance of point-of-care ultrasound is increasingly recognized among critical patients, it is emerging as an important therapeutic and diagnostic tool in obstetric anesthesia.
\end{abstract}

Volume 8 Issue 4 - 2019

\author{
Ahmed Altraigey,' Samar Rafik ${ }^{2}$ \\ 'Department of Obstetrics and Gynaecology, Benha University, \\ Egypt \\ ${ }^{2}$ Department of Anaesthesia, Benha University, Benha, Egypt
}

Correspondence: Ahmed Altraigey MD, Department of Obstetrics and Gynaecology, Benha University, 43 BenhaZagazig Street, Mansheyet Elnoor, Benha, I35 I I, Arab Republic of Egypt, Egypt, Tel +201010137855,

Email ahmed.abdelfattah@fmed.bu.edu.eg

Received: May 08, 2019 | Published: July 22, 2019

\section{Introduction}

Ultrasound has gained a valuable position in anesthetic practice; "unblinding" many procedures that were once "blind". What made Ultrasound (US) such a useful diagnostic and monitoring option in medicine is being portable, inexpensive, safe and easily accessible imaging equipment. Appearance of ultrasound use in clinical anesthesia was firstly described almost forty years ago and its role in obstetric anesthesia draw attention following a series of publications in the early 2000s. Meanwhile, the most popular applications in anesthetic practice were peripheral nerve block guided by ultrasound and establishment of vascular access. ${ }^{1}$

Nowadays, Ultrasound is expanding its role to the perioperative management via Point-of-Care Ultrasound (POCUS). The concept of POCUS is a much more recent development, it is defined as "a diagnostic or procedural ultrasound guidance that is performed at the bed side by a clinician to obtain rapid evaluation or treatment of the patient". ${ }^{2}$ Being the " $21^{\text {st }}$-century stethoscope", POCUS has been described because it is a non- invasive method using images to give the traditional bedside examination, more meaningful clinical data. Recently, many anesthesiology subspecialties, such as critical care, regional anesthesia, pediatrics and obstetrics are pushed to introduce POCUS skills to their practice. ${ }^{3}$ As a consequence, there has been a "call to action" to encourage perioperative (US) use by all anesthesiologists through establishment of an organized educational training program added to the curriculum of anesthesia trainees. ${ }^{4}$

POCUS has numerous applications in clinical anesthesia including: focused cardiac ultrasound (FoCUS) or transthoracic echocardiography (TTE), airway, lung, brain, abdominal and gastric assessment, evaluation of deep venous thrombosis, intracranial tension measurement, ultrasound-guided maneuvers such as regional anesthesia, vascular and airway access, drainage of pericardial and pleural effusions, monitoring adequacy of recruitment maneuvers and assessment of splanchnic perfusion, etc. The applications are growing and developing, with the concept of whole body point-ofcare ultrasound starting to be introduced into the anesthesiologist's skill program. ${ }^{5}$

\section{Review of literature}

Central neuraxial blockade is the most common anesthetic method utilized in obstetric anesthesia. As a result, the application of ultrasound in the practical field expanded its interest from peripheral nerve blocks to make spinal/epidural procedures easier in obstetrics. Nowadays, as higher quality ultrasound machines have become more available and inexpensive, so their popularity has risen further as a technique for diagnosis and procedures at bedside. ${ }^{6}$ The majority of peripartum women that anesthesiologists involved with, are at low risk for developing complications, but still the possibility for serious scenarios that threaten the health and life of both the mother and her fetus can occur. These may happen in a low-risk unit, and can rapidly develop, making it difficult to secure a specialist for diagnostic help. Invasive monitoring is not preferred and irradiation is only used when fundamental due to its risks to the fetus. Ultrasound is available in obstetric practice for fetal monitoring. It also has the ability for bedside screening and diagnostic procedures of the mother, as an extension of clinical evaluation. Pregnancy is associated with increased risk of difficult airway access, aspiration of stomach contents, acute pulmonary failure and cardiovascular decompensation. ${ }^{7}$

This review covers the use of ultrasound in emergency obstetric anesthesia management to assess difficult airways via identifying the cricothyroid membrane, to avoid rapid sequence induction if an empty stomach can be confirmed, to identify the insertion point for a rapid spinal for emergency cesarean delivery and for assessment of shock during pregnancy. 


\section{Airway management}

The incidence of failed intubation in pregnant women has been reported as 2.3 per 1000 general anesthetics for caesarean section, and 2.6 per 1000 general anesthetics for obstetric procedures. ${ }^{8}$ In spite of using epidural analgesia early in labor to avoid the need for general anesthesia if urgent cesarean delivery is required, this strategy does not always guarantee successful surgical anesthesia especially in obese women who are subjected more to the risk of failed epidurals and failed intubation. Hence, The Obstetric Anesthesia Airway guidelines recommend a strong antenatal plan for women with predicted difficult airway. ${ }^{9}$ If failed tracheal intubation scenario occurred in pregnant woman, general anesthesia is usually continued rather than awakening the patient. The last guidelines of Obstetric Anaesthetists' Difficult Airway Society for the management of difficult and failed tracheal intubation in obstetric recommend conducting an airway evaluation before initiation of general anesthesia. This evaluation helps to expect any difficulty in the management of airway including mask ventilation, tracheal intubation, supraglottic device usage and frontof-neck airway access. ${ }^{9}$ Hui et al..$^{9}$ used ultrasound to predict difficult airway by imaging the sublingual space in a non-pregnant population, by placing the curved probe, covered with a sterile sheath, under their tongue in a longitudinal plane. Inability to visualize the hyoid bone was a sign to predict difficult intubation, and this technique may be applied in pregnant women too. ${ }^{10}$ Identification of the position of cricothyroid membrane has a great importance in the difficult airway management and failure to locate it may lead to a failed invasive airway procedure. Kinsella et al., ${ }^{8}$ revealed that one in 60 women who failed to be intubated may require surgical airway access, and knowledge of how to perform these techniques may be life-saving to such patients. ${ }^{8}$

For ultrasound identification of the cricothyroid membrane, the transverse and longitudinal techniques have been described. The transverse technique identifies the cricothyroid membrane as a hyperechoic line seen between the cricoid cartilage caudal and the thyroid cartilage cephalad by placing the probe transversely on the neck. The longitudinal technique view the tracheal rings as "string of pearls" by placing the probe transversely initially, then rotated $90^{\circ}$ into the sagittal plane, and then cephalad until the cricoid cartilage and the thyroid cartilage are identified and the membrane in between, ${ }^{11}$ It is difficult to locate the cricothyroid membrane using digital palpation, especially in obese non-pregnant and laboring women. You-Ten et al. ${ }^{12}$ compared ultrasound-guided versus digital palpation of cricothyroid anatomy in 56 women (half with BMI $>30 \mathrm{~kg} / \mathrm{m} 2$ ). They found that ultrasound improved locating the membrane, and this was of great help when used among obese women with short neck. ${ }^{12}$

Point-of-care airway ultrasound also shows a benefit to confirm the correct placement of the tube in the trachea. Ultrasound examination of the trachea during laryngoscopic intubation, using a transverse probe, permits visualization of correct tube placement as a hyperechoic shadow in the trachea in real-time. Conversely, enlargement of the oesophagus indicates for oesophageal intubation. ${ }^{13}$

\section{Gastric volume assessment}

For obstetric general anesthesia, rapid sequence induction and tracheal intubation has traditionally been recommended, because of the potential risk of regurgitation and lung aspiration in unfasted women presenting for emergency procedures, as well as decreased tone of the lower esophageal sphincter during pregnancy. It is documented that second- and third-trimester pregnant women is considered to have a 'full stomach', that is, above the risk threshold for gastric contents aspiration. Gastric ultrasound allows evaluation of the volume and type of the stomach contents; this provides an accurate detection of the hazards and help making a proper decision. ${ }^{14}$

The technique of the gastric antrum examination via Point-of-care ultrasound has been standardized in non-pregnant adults. Using a $2-5-\mathrm{MHz}$ curved probe in the epigastric area, the gastric antrum is recognized in the sagittal plane, identified by the left hepatic lobe and the aorta as landmarks. The antrum is considered empty if it is flat, with anterior and posterior walls in close contact to each other. When antrum is dilated with hypo-echoic matter, this indicates presence of clear fluid contents. While solid contents are indicated by viewing an echoic matter. ${ }^{15}$ Perlas et al. ${ }^{16}$ described a 3 -grade grading scale that is frequently used to describe the gastric volume. Grade 0 , when an empty antrum is seen in both right lateral decubitus and supine positions, this indicates absence of any contents. Grade 1, when seeing fluid content only in the right lateral decubitus position, this usually corresponds to a stomach fluid volume of $<100 \mathrm{ml}$ in $77 \%$ of patients. Grade 2, when seeing fluid contents in both supine and right lateral decubitus positions, this corresponds to a stomach fluid volume $>100$ $\mathrm{ml}$ in $75 \%$ of patients as well $>250 \mathrm{ml}$ volume in $50 \%$ of patients. At third grade a potential risk for aspiration is associated. ${ }^{16}$

The gastric antrum examination using ultrasound is more challenging in pregnant and laboring women than in non-pregnant women, with a rate of doubt that may reach $17.4 \%$. This may be owing to epigastric probe location may be inhibited by the steep angle between the xiphoid process and abdomen due to the enlarged gravid uterus. Upward displacement of the stomach by the gravid uterus obscure ultrasound views of the stomach antrum via the left hepatic lobe and the aorta in the sagittal plane. The antrum depth, the space between the stomach antrum and the aorta and the antral crosssectional area increases during pregnancy significantly. ${ }^{17}$

Other mathematical methods were introduced for quantity assessment of gastric volume as an alternative to classic grad. Measurement of the gastric antrum cross-sectional area via ultrasound view in the sagittal plane through estimating the maximum anteroposterior (D1) and longitudinal (D2) diameters, then calculates antral cross-sectional area through a special mathematical equation. The antral cross-sectional area proportionate directly or indirectly with measured gastric volume. ${ }^{18}$ Bouvet el al. ${ }^{19}$ suggested measuring antral cross-sectional area in the semirecumbent position to differentiate between gastric contents above or below a risk threshold which estimated as (gastric volume $>0.8 \mathrm{ml} / \mathrm{kg}$ ) or (presence of solid contents). However, by using a formula that contains the age of the patient, the right lateral decubitus position is found to be more dependable than the semirecumbent position in obtaining gastric fluid volume through antral cross-sectional area. ${ }^{19}$

Arzola et al., ${ }^{20}$ estimated volumes of gastric contents in 103 pregnant women before elective cesarean delivery under neuraxial blockade. There was agreement in measures of Gastric antral area between the supine and the lateral decubitus position in $95 \%$ of the patients. expected gastric volumes in this cohort study in pregnant women corresponded to actual ingestion of Grade $0=0.4 \mathrm{ml} / \mathrm{kg}$, Grade $1=1.0 \mathrm{ml} / \mathrm{kg}$, and Grade $2=2.7 \mathrm{ml} / \mathrm{kg}$, and these results were resemble to volumes reported in the non-pregnant women. ${ }^{20}$

There are incompatible publications concerning identification of an empty stomach in gravid women. Arzola et al..$^{20}$ recognized in his cohort that the stomach volume could be confirmed reliably, 
opposing other previous studies in pregnant women. This difference in results can be explained by the development in technology. There are other restrictions to utilize gastric ultrasound. First, the effect of neuraxial blockade on stomach volume is not obvious, and the pregnant women included in the studies had prolonged fasting time. In addition to difficulty in techniques to identify the antrum, the measure of D1 and D2 may be inaccurate in pregnancy, as the stomach is pushed cephalad. Although measures of stomach volume in the lateral decubitus position may be more accurate, supine position may be necessary in emergency situations. A failure rate between 13 and $36 \%$ has been reported to achieve a good scan. Finally, lack of training in gastric ultrasound assessment to anesthesiology residents should be considered. At least 33 scans were required for competency in gastric ultrasound training, to achieve a success rate of $95 \%,{ }^{21}$ Furthermore, measures reported in the studies done by expert operators, which may not be applicable in a clinical situation. It is still too early to predict the efficacy of ultrasound to detect gastric contents, to establish clinical decisions on aspiration risk, and to plan airway management for general anesthesia. Although current studies lack reliability with clinical decision-making depending on measured gastric volumes, but improving training and increase familiarity may come in the future with more reliable results. ${ }^{7}$

\section{Neuraxial blocks}

It is challenging in pregnant women to establish neuraxial blockade depending on the traditional techniques of landmarks assessment, owing to the anatomical and physiological changes such as obesity, edema, lordosis, scoliosis and difficult positioning. The estimated incidence of difficult neuraxial block seems to be around $4 \%{ }^{22}$

According to Stendell et al., ${ }^{22}$ unrecognizable landmarks was the most frequent sign for difficult neuraxial blockade among 73579 patients; operator experience is also important. The more difficulty to establish the blocks, the higher the frequency of complications produced; such as inadvertent dural puncture, backache, paresthesia and other neurological sequelae. ${ }^{22}$ In addition, proper positioning of the patient to facilitate the block can be challenging because of the gravid uterus. In 2016, a publication based on two meta-analyses reported that pre-procedural use of ultrasound decreased the number of trials needed to achieve the block compared with traditional palpation of landmarks. ${ }^{23}$ However, there were different finding across all patient populations, as demonstrated by Tawfik et al., who failed to obtain such advantage when comparing a neuraxial block using ultrasound with traditional landmark palpation in a non-obese $(\mathrm{BMI}<35 \mathrm{~kg} /$ $\mathrm{m} 2$ ) full-term singleton pregnancy population undergoing elective cesarean delivery. ${ }^{24}$

The pitfalls of ultrasound include consuming time to perform the procedure while the painful labor presents. In one study, using ultrasound increased the duration of the neuraxial procedure by $75 \mathrm{~s}$ in a population of 75 laboring women, versus same number of women in whom the block was performed using traditional palpation. ${ }^{25}$ This may be a valuable time when a woman is in need for immediate analgesia or anesthesia in labor; moreover, in the study setting, the staff were aware of the study and had experience in ultrasound usage, this helped the time to be optimally short. Arzola ${ }^{20}$ emphasized that many physicians may avoid dealing with ultrasound due to the lack of technical experience. neuraxial block assisted ultrasound require regular training to fasten the block placement in difficult cases such as patients with scoliosis, obesity, etc. ${ }^{23}$ Current evidence suggests that when anesthesiologist predicts a difficult neuraxial block for labor, using ultrasound assistance to identify the landmarks will improve the efficacy to perform the block with fewer punctures. It is attractive to imagine being able to visualize the needle journey through the anatomical structures till it ends in the epidural space in real time. ${ }^{6}$

\section{Diagnosis of shock during pregnancy}

Shock a life-threatening condition which is defined as failure of adequate oxygen delivery to the tissues due to either inadequate blood supply to tissues, decreased blood oxygen saturation, or increased tissues oxygen demand that results in end-organ dysfunction. ${ }^{26}$ Maternal shock during pregnancy often is caused by bleeding, however it can occur secondary to other pathological conditions such as (septic infection, idiopathic peripartum cardiomyopathy, amniotic fluid embolism, pulmonary embolism, myocardial infarction). In such intensive and emergency conditions, bed-side point-of-care ultrasound can affect the diagnosis and treatment of shock states considerably, since sonographic findings are easily apparent and usually significant. ${ }^{27}$

\section{Obstructive shock}

The most common cause of obstructive shock is pulmonary embolism that obstruct left ventricular filling, whereas the rarer causes like tension pneumothorax and cardiac tamponade obstruct right ventricular filling. Tension pneumothorax is manifested by rapid deterioration of respiration, associated with asymmetrical clinical findings upon chest examination. In the absence of subcutaneous emphysema, point-of-care ultrasound is significant and superior to chest X-ray for diagnosis of pneumothorax. ${ }^{28}$

Ultrasound images show pneumothorax as an anterior disappearance of normal lung sliding, with a horizontal A-line. Moving the probe to the lateral side of the chest wall can visualize the point where lung sliding recurs ('lung point'). The presence of a lung point is pathognomonic of pneumothorax and should initiate immediate draining of the excess air. Cardiac tamponade is defined as a collection of fluid in the pericardial sac, leading to compression of cardiac chambers. ${ }^{29}$ Many causes can lead to cardiac tamponade including spontaneous pericardial effusion, trauma or aortic dissection. Its occurrence during pregnancy or peripartum is due to the combined hormone-induced changes in connective tissue and haemodynamic stress. Image diagnosis is simply done by using a sub costal or parasternal long-axis view. If a circumferential effusion $>1$ $\mathrm{cm}$, or right chamber compression associated with shock, an Urgent surgical consultation should be obtained. ${ }^{30}$

Pulmonary embolism can occur during pregnancy or the postpartum period. When it is associated with shock, point-of-care ultrasound will show signs of right ventricular dilation with flattening or paradoxical septal motion. An assessment of the venous system of the lower limbs (the femoral and popliteal regions) should be examined by the ultrasound as well, searching for incompressible veins or intraluminal thrombus. ${ }^{31}$

\section{Cardiogenic shock}

An essential tool for evaluation of left ventricular function is echocardiography, using visual estimation of a short-axis or apical 4-chamber view. ${ }^{32}$ Cardiac ultrasound (Echocardiography) is recommended to be used for haemodynamic evaluation in preeclamptic patients. Myocardial dysfunction may be developed due to late-stage pre-eclampsia-related heart failure or peripartum cardiomyopathy; their risk can extend not only during pregnancy but also for period afterwards. ${ }^{33}$ 
Improper management of myocardial infarction can cause regional myocardial wall motion abnormality which in turn can progress to cardiogenic shock. Myocardial infarction in the postpartum period holds three times risk as that of non-pregnant women and echocardiography represents the cornerstone of its diagnosis and monitoring. ${ }^{34}$

\section{Hypovolemic and distributive shock}

Hemorrhage is the most common cause for hypovolemic shock in the peripartum period that should initially be assumed, unless there are clear signs of sepsis or severe dehydration. Hypovolemia can be diagnosed using Point-of-care ultrasound even in the absence of apparent external bleeding. 'Kissing heart' is a sensitive and easily detectable sign of hypovolemia, in which the mitral pillars collapse during left ventricle systole. This is associated with a very low left ventricular diastolic filling volume. ${ }^{32}$ In sever hypovolemia; the subcostal longitudinal view may show the image of "kissing heart" associated with inspiratory collapse of the inferior vena cava. An abdominal ultrasound assessment should always be performed in the presence of shock, in order to search for evidence of free intraperitoneal fluid or fluid outside the Douglas pouch which raise suspicion. ${ }^{35}$ Distributive shock results from excessive vasodilation and subsequent impaired distribution of blood flow. Most commonly caused by sepsis or anaphylaxis wich present similar to hypovolemic shock with kissing heart sign and inferior vena cava collapse. But left ventricular ejection fraction is normal or increased in turn a high cardiac output is present. This is considered a discriminating sign between hypovolemic and distributive shock. ${ }^{7}$

\section{Acknowledgments}

None.

\section{Conflicts of interest}

Authors declare that there is no conflict of interest.

\section{Funding}

No.

\section{References}

1. Terkawi AS, Karakitsos D, Elbarbary M, et al. Ultrasound for the anesthesiologists: present and future. The scientific world journal. 2013;2013(1):1-15.

2. Haskins SC, Vaz AM, Garvin S. Perioperative Point-of-Care Ultrasound for the Anesthesiologist. Journal of Anesthesia and Perioperative Medicine (JAPM). 2018;28;5(2):92-96.

3. Haskins SC, Boublik J, Wu CL. Point-of-Care Ultrasound for the Regional Anesthesiologist and Pain Specialist: A Series Introduction. Reg Anesth Pain Med. 2017;42(3):281-282.

4. Mahmood F, Matyal R, Skubas N, et al. Perioperative Ultrasound Training in Anesthesiology: A Call to Action. Anesth Analg. 2016;122(6):1794804 .

5. McCormick TJ, Miller EC, Chen R, et al. Acquiring and maintaining point-of-care ultrasound (POCUS) competence for anesthesiologists. Canadian Journal of Anesthesia/Journal canadien d'anesthésie. 2018;65(4):427-436.

6. Weiniger CF, Sharoni L. The use of ultrasound in obstetric anesthesia. Current Opinion in Anesthesiology. 2017;30(3):306-312.

7. Zieleskiewicz L, Bouvet L, Einav S, et al. Diagnostic point-ofcare ultrasound: applications in obstetric anaesthetic management. Anaesthesia. 2018;73(10):1265-1279.
8. Kinsella SM, Winton AL, Mushambi MC, et al. Failed tracheal intubation during obstetric general anaesthesia: a literature review. Int $J$ Obstet Anesth. 2015;24(4):356-374.

9. Mushambi MC, Kinsella SM, Popat M, et al. Obstetric Anaesthetists' Association and Difficult Airway Society guidelines for the management of difficult and failed tracheal intubation in obstetrics. Anaesthesia. 2015; 70(11):1286-1306.

10. Hui CM, Tsui BC. Sublingual ultrasound as an assessment method for predicting difficult intubation: a pilot study. Anaesthesia. 2014;69(4):314-319.

11. Kristensen MS. Ultrasonography in the management of the airway. Acta Anaesthesiologica Scandinavica. 2011;55(10):1155-1173.

12. You-Ten KE, Desai D, Postonogova T, et al. Accuracy of conventional digital palpation and ultrasound of the cricothyroid membrane in obese women in labour. Anaesthesia. 2015;70(11):1230-1234.

13. Lucas DN, Elton CD. Through a glass darkly-ultrasound imaging in obstetric anaesthesia. Anaesthesia. 2016;71(6):617-622.

14. Van de Putte P, Perlas A. The link between gastric volume and aspiration risk. In search of the Holy Grail? Anaesthesia. 2018;73(3):274-279.

15. Bouvet L, Chassard D. Contribution of ultrasonography for the preoperative assessment of gastric contents. Annales Francaises D'Anesthesie et de Reanimation. 2014;33(4):240-247.

16. Perlas A, Mitsakakis N, Liu L, et al. Validation of a mathematical model for ultrasound assessment of gastric volume by gastroscopic examination. Anesthesia and Analgesia. 2013;116(2):357-363.

17. Rouget C, Chassard D, Bonnard C, et al. Changes in qualitative and quantitative ultrasound assessment of the gastric antrum before and after elective caesarean section in term pregnant women: a prospective cohort study. Anaesthesia. 2016;71(11):1284-1290.

18. Zieleskiewicz L, Boghossian MC, Delmas AC, et al. Ultrasonographic measurement of antral area for estimating gastric fluid volume in parturients. Br J Anaesth. 2016;117(2):198-205.

19. Bouvet L, Mazoit JX, Chassard D, et al. Clinical assessment of the ultrasonographic measurement of antral area for estimating preoperative gastric content and volume. Anesthesiology. 2011;114(5):1086-1092.

20. Arzola C, Perlas A, Siddiqui NT, et al. Bedside gastric ultrasonography in term pregnant women before elective cesarean delivery: a prospective cohort study. Anesth Analg. 2015;121(3):752-758.

21. Arzola C, Cubillos J, Perlas A, et al. Interrater reliability of qualitative ultrasound assessment of gastric content in the third trimester of pregnancy. Br J Anaesth. 2014;113(6):1018-1023.

22. Stendell L, Lundstrom LH, Wetterslev J, et al. Risk factors for and prediction of a difficult neuraxial block: a cohort study of 73579 patients from the Danish anaesthesia database. Reg Anesth Pain Med. 2015;40(5):545-552.

23. Arzola C. Preprocedure ultrasonography before initiating a neuraxial anesthetic procedure. Anesth Analg. 2017;124(3):712-713.

24. Tawfik MM, Atallah MM, Elkharboutly WS, et al. Does preprocedural ultrasound increase the first-pass success rate of epidural catheterization before cesarean delivery? A randomized controlled trial. Anesth Analg. 2017;124(3):851-856.

25. Ansari T, Yousef A, El Gamassy A, et al. Ultrasound-guided spinal anaesthesia in obstetrics: is there an advantage over the landmark technique in patients with easily palpable spines? Int J Obstet Anesth. 2014;23(3):213-216.

26. Cecconi M, De Backer D, Antonelli M, et al. Consensus on circulatory shock and hemodynamic monitoring. Task force of the European Society of Intensive Care Medicine. Intensive Care Medicine. 2014;40(12): 1795-1815. 
27. Zieleskiewicz L, Muller L, Lakhal K, et al. Point-of-care ultrasound in intensive care units: assessment of 1073 procedures in a multicentric, prospective, observational study. Intensive Care Medicine. 2015;41(9):1638-1647.

28. Xirouchaki N, Magkanas E, Vaporidi K, et al. Lung ultrasound in critically ill patients: comparison with bedside chest radiography. Intensive Care Medicine. 2011;37(9):1488-1493.

29. Klein AL, Abbara S, Agler DA, et al. American Society of Echocardiography clinical recommendations for multimodality cardiovascular imaging of patients with pericardial disease. Journal of the American Society of Echocardiography. 2013;26(9):965-1012.

30. European Society of Gynecology (ESG), Association for European Paediatric Cardiology (AEPC), German Society for Gender Medicine (DGes GM), et al. ESC Guidelines on the management of cardiovascular diseases during pregnancy: the Task Force on the Management of Cardiovascular Diseases during Pregnancy of the European Society of Cardiology (ESC). European Heart Journal. 2011;32(4):3147-3197.
31. Nazerian P, Vanni S, Volpicelli G, et al. Accuracy of pointof- care multiorgan ultrasonography for the diagnosis of pulmonary embolism. Chest. 2014;145(5):950-957.

32. Porter TR, Shillcutt SK, Adams MS, et al. Guidelines for the use of echocardiography as a monitor for therapeutic intervention in adults: a report from the American Society of Echocardiography. Journal of the American Society of Echocardiography. 2015;28(1):40-56.

33. Dennis AT. Transthoracic echocardiography in women with preeclampsia. Current Opinion in Anesthesiology. 2015;28(3):254-260.

34. James AH, Jamison MG, Biswas MS, et al. Acute myocardial infarction in pregnancy. A United States population-based study. Circulation. 2006;113(12):1564-1571.

35. Sentilhes L, Vayssiere C, Deneux-Tharaux C, et al. Postpartum hemorrhage: guidelines for clinical practice from the French College of Gynaecologists and Obstetricians (CNGOF). European Journal of Obstetrics \& Gynecology and Reproductive Biology. 2016;198:12-21. 